\title{
Minimum Rate Guaranteed Call Admission Control for Cumulative Rate Distribution Based Scheduling
}

\author{
Hao Wang, Lianghui Ding, Nan Liu, Zhiwen Pan, Ping Wu, and Xiaohu You, Senior Member, IEEE
}

\begin{abstract}
In this letter, we investigate call admission control (CAC) for cumulative rate distribution based scheduling (CS) in wireless communication networks. We first analyze the multiuser diversity gain (MDG) of CS, which is applicable to general channel conditions. Then we propose the minimum rate guaranteed CS/ORR based CAC algorithm, COCAC, which jointly uses $\mathrm{CS}$ and opportunistic round robin (ORR) for resource prediction and $\mathrm{CAC}$ decision. Furthermore, we evaluate the performance of our COCAC algorithm through simulation. Results show that COCAC can significantly reduce the new call blocking rate, while strictly guarantee the minimum rate requirements of all serving users.
\end{abstract}

Index Terms-Call admission control (CAC), cumulative rate distribution based scheduling (CS), multi-user diversity gain (MDG), opportunistic round robin (ORR).

\section{INTRODUCTION}

$\mathbf{O}$ PPORTUNISTIC scheduling has been widely applied in wireless networks to utilize the dynamic channel variations of different users. It can achieve the multi-user diversity gain (MDG), which is defined as the ratio between the long-term average throughput of opportunistic scheduling and that of round robin scheduling (RRS). However, theoretically analyzing the MDG of opportunistic scheduling under general channel conditions is still an open question. For existing opportunistic scheduling schemes, only the MDG of proportional fairness scheduling (PFS) [1] has been derived in [2] by assuming independent and identically distributed (i.i.d.) channels among users. In PFS, the user with the highest ratio between the instantaneous rate and the achieved throughput is scheduled. Thus, it is difficult to derive the MDG of PFS in practical scenarios with non i.i.d. channels, which may result from distinct location and surroundings of each user. In contrast, cumulative rate distribution based scheduling (CS) schedules the user with the maximum cumulative rate distribution, and allocates almost equal resources to each user, which is not affected by the heterogeneity of channels among users [3], [4]. However, there is still a lack of analytical result on the long-term average performance of CS. Thus our first

Manuscript received June 8, 2011. The associate editor coordinating the review of this letter and approving it for publication was I.-R. Chen.

H. Wang, N. Liu, Z. Pan, and X. You are with the National Mobile Communications Research Laboratory, Southeast University, Nanjing, China (e-mail: \{hao_wang, nanliu, pzw, xhyu\}@seu.edu.cn).

L. Ding is with the Dept. of Electronic Engineering, Shanghai Jiao Tong University, Shanghai, China (e-mail: lhding@ ieee.org).

$\mathrm{P}$. Wu is with the Dept. of Engineering Sciences, Uppsala University, Uppsala, Sweden (e-mail: ping.wu@angstrom.uu.se).

This work is supported by the International Science and Technology Cooperation Program under grant 2008DFA12090; the National Mobile Communications Research Laboratory Program (2010A02, 2011A02); the National Special Key Program (2011ZX03003-002-02); and Huawei Corp. Ltd.

Digital Object Identifier 10.1109/LCOMM.2011.082911.111134 contribution in this letter is deducing the MDG of CS. Thanks to the special resource allocation policy of CS, the derived MDG of CS can be applied to wireless networks with general channel distributions.

A promising character of the next-generation networks is to serve as many users as possible with strict guarantee of quality of service (QoS). Thus, it is important to use call admission control (CAC) to control the number of admitted users according to the amount of available resources. Although minimum rate and average delay are two important QoS metrics in next-generation networks, the second one can be potentially guaranteed by the minimum rate requirement (MRR) [5]. Hence, for mathematical tractability, we only consider MRR as the QoS metric in the following analysis. In a real system, users usually have heterogeneous MRRs, thus the MDG derived from fair resource allocation cannot be directly used to make the admission decision for a new user. Therefore, as the second contribution of this letter, we propose a minimum rate guaranteed CS/ORR based CAC algorithm, COCAC, which jointly uses CS and opportunistic round robin (ORR) [6] to conservatively estimate the resource occupation of new access users and make the CAC decision.

Extensive simulation is then conducted to evaluate the performance of our proposed COCAC algorithm in terms of new call blocking rate and MRR violation ratio.

\section{Multi-user Diversity Gain of CS}

First we briefly review the CS scheme [3]. The main idea of $\mathrm{CS}$ is to schedule the user with the maximal cumulative rate distribution in each token, i.e., the basic unit for scheduling. Let $R_{k}, f_{R_{K}}$ and $F_{R_{K}}$ denote the random variables of user $k$ 's rate, Probability Density Function (PDF) of $R_{k}$ and Cumulative Distribution Function (CDF) of $R_{k}$, respectively. Let $r_{k}$ denote the instantaneous value of $R_{k}$ in a token, then the user $k^{*}$ with the highest $F_{R_{k^{*}}}\left(r_{k^{*}}\right)$ is selected for communication. Assuming there are $n$ competing users, CS can be written as

$$
k^{*}=\arg \max _{1 \leq k \leq n} F_{R_{k}}\left(R_{k}\right)
$$

Let $U_{k}$ denote the random variable $F_{R_{k}}(\cdot)$, which is uniformly distributed in $[0,1]$, and there exists $F_{R_{k}}\left(r_{k}\right)=u_{k}$. The probability that user $k$ is chosen for communication is

$$
\begin{aligned}
\operatorname{Pr}\left(k^{*}=k\right) & =\operatorname{Pr}\left(U_{j} \leq u_{k}, \text { for all } j \neq k\right) \\
& =\prod_{j=1, j \neq k}^{n} F_{U_{j}}\left(u_{k}\right) \\
& =u_{k}^{n-1}
\end{aligned}
$$


Hence, the probability for user $k$ to be selected is $\int_{0}^{1} u_{k}^{n-1} d u_{k}=1 / n$, which indicates that resources are allocated to users fairly. Without loss of generality, we assume $F_{R_{k}}(\cdot)$ is a strict increasing continuous function, and its inverse is $F_{R_{k}}^{-1}(\cdot)$. Then the rate distribution seen by user $k$ in all tokens is the same as $F_{R_{k}}^{-1}\left(U_{k}\right)$. Therefore, the long-term average throughput of user $k$ in CS is

$$
\begin{aligned}
T_{n, k} & =\int_{0}^{1} \operatorname{Pr}\left(k^{*}=k\right) F_{R_{k}}^{-1}\left(u_{k}\right) d u_{k} \\
& =\int_{0}^{1} u_{k}^{n-1} F_{R_{k}}^{-1}\left(u_{k}\right) d u_{k}
\end{aligned}
$$

Let $D_{n, k}$ denote user $k$ 's long-term average throughput in RRS, it can be written as

$$
D_{n, k}=\frac{1}{n} \int_{0}^{+\infty} x_{k} f_{R_{k}}\left(x_{k}\right) d x_{k}
$$

where $x_{k}$ is the instantaneous rate of user $k$, and $f_{R_{k}}\left(x_{k}\right)$ is the PDF of $x_{k}$.

Then the MDG of CS for user $k$, which uses RRS as the benchmark, is defined as

$$
G_{n, k}^{C S}=\frac{T_{n, k}}{D_{n, k}}
$$

It is easy to see that $G_{n, k}^{C S}$ is determined by the number of competing users and the distribution of $R_{k}$, while independent of the rate properties of other users. When reasonably assuming Rayleigh fast fading and a linear rate to SINR dependency for all users, $R_{k}(1 \leq k \leq n)$ are exponentially distributed, and $G_{n, k}^{C S}$ can be simplified into

$$
G_{n, k}^{C S}=\sum_{i=1}^{n} \frac{1}{i}
$$

which indicates that, with the above two assumptions, the MDG of CS for any user $k$ only depends on the number of competing users. Thus, we can use $G_{n}^{C S}$ instead of $G_{n, k}^{C S}$ in the following analysis for convenience.

\section{Minimum Rate Guaranteed CS/ORR based Call ADMISSION CONTROL}

To admit a new user with MRR to the network, it is important to decide whether the residual resources are enough to satisfy the MRR of the new user without impacting all existing ones. In practice, users usually have different MRRs and average SINRs, thus the quantity of resources they required are also different from each other, and the MDG derived above for fair resource allocation cannot be directly used to calculate the amount of resource needed by the new user. On the other hand, ORR has been proved to be an effective method to calculate the statistical lower bound on performance of any opportunistic scheduling scheme [7]. Thus, in this letter, we jointly use CS and ORR to conservatively estimate the resource needed for the new user.

Resources are allocated to users round by round in CS/ORR. In each round, tokens equal to the number of existing users are scheduled and each user competes for resources through CS. All users achieving their MRRs in a round will quit the resource allocation process in the following rounds. In one round, once a user has been served and gotten a token, it will not compete for the residual tokens. Thus, for $n$ users in a round, the MDG for the first served user is $G_{n}^{C S}$, that for the second one is $G_{n-1}^{C S}, \ldots$, and that for the last one is $G_{1}^{C S}$. Therefore, the average MDG of CS/ORR $G_{n}^{C O}$ in that round can be written as

$$
G_{n}^{C O}=\frac{1}{n} \sum_{l=1}^{n} G_{l}^{C S}=\frac{1}{n} \sum_{l=1}^{n} \sum_{i=1}^{l} \frac{1}{i}
$$

Since admitting a new user will give more chances to all existing ones to exploit opportunism [7], we only need to consider whether the residual tokens can satisfy the MRR of the new user through CS/ORR scheduling. We assume that the set of serving users is $\mathbf{Z}$, and there have been $n$ users with MRRs $r_{1}, \ldots, r_{n}$ and average data rates $\lambda_{1}, \ldots, \lambda_{n}$ in $\mathbf{Z}$. We also assume the scheduling is executed periodically and the residual tokens in last period is $t_{\text {res }}$. When a new user $m$ with MRR $r_{m}$ and average data rate $\lambda_{m}$ asks to enter the system, we perform the following CAC algorithm,

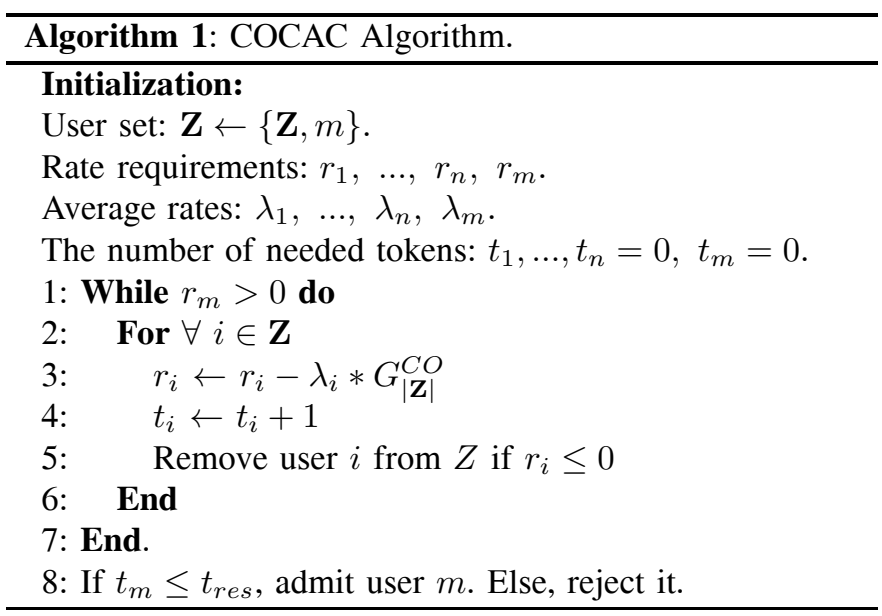

Remark 1. The above algorithm is not limited to CS. For any scheduling method with a closed-form expression of $M D G G^{*}$, our algorithm still works by replacing $G_{l}^{C S}$ in (7) with $G^{*}$.

\section{Simulation And Numerical Results}

\section{A. Simulation Setup}

We assume Rayleigh fast fading, and the feedback of channel states is error free and has no delay. We use Shannon capacity as the communication rate for theoretical analysis, while ignoring the errors from practical modulation and coding schemes. All users arrive according to a Poisson process with rate $\lambda$ and depart from the system after a holding time that is exponentially distributed with the mean of 100 seconds. SINR and MRR of each user are normally distributed in $[0,10] \mathrm{dB}$ and in $[50,100] \mathrm{kbps}$, respectively. The size of scheduling token is consistent with the definition of a physical resource block in 3GPP LTE, i.e., $180 \mathrm{kHz} \times 1 \mathrm{~ms}$. The scheduling period is one second which contains 5000 tokens and 1000 scheduling periods are considered in each simulation. For each user arrival rate, the simulation is conducted 100 times, and the average performance is given. 


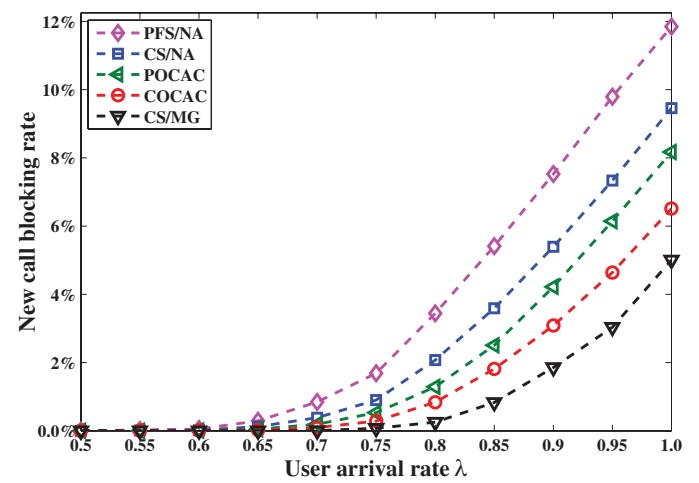

Fig. 1. New call blocking rate.

The performance of our proposed COCAC algorithm is evaluated in terms of new call blocking rate and MRR violation ratio. In the following, we use PFS/NA, CS/NA, $C S / M G$ and POCAC to represent PFS with no MDG, CS with no MDG, CS with maximum MDG and PFS with ORR, respectively. Here, no MDG and maximum MDG mean that the needed tokens are calculated via RRS and by (6), respectively, i.e., our CAC algorithm is performed by replacing $G_{|\mathbf{Z}|}^{C O}$ with 1 and $G_{|\mathbf{Z}|}^{C S}$, respectively. $P O C A C$ is similar to COCAC, which jointly uses ORR and the MDG of PFS in [2] for CAC. Users in PFS/NA and POCAC are scheduled through PFS, while those in $C S / N A, C S / M G$ and COCAC are scheduled through CS. Once a user having achieved its MRR, it quits the scheduling process. When all existing users have achieved their MRRs, the residual tokens, the number of which is $t_{r e s}$, are scheduled to all users by using corresponding scheduling schemes. Thanks to the time share fairness property of CS, residual tokens in $C S / N A, C S / M G$ and COCAC can be allocated to all users fairly.

\section{B. Simulation Results}

1) New Call Blocking Rate: As shown in Figure 1, the new call blocking rates in all cases increase monotonously with the user arrival rate. This is reasonable because that the larger the arrival rate, the more the users to arrive and be blocked due to resource limitation. The new call blocking rate of POCAC is less than that of PFS/NA because of the MDG of PFS used in POCAC. The new call blocking rates of both COCAC and $C S / M G$ are less than that of CS/NA due to the multi-user diversity considered in COCAC and $C S / M G$. Moreover, the new call blocking rate of $C S / M G$ is smaller than that of COCAC, because $C S / M G$ admits more users by using the aggressive MDG of CS in (6) ignoring heterogeneous resource demands of different users. The consequence is that $C S / M G$ has a higher MRR violation ratio as shown in Figure 2.

The curves of CS/NA and COCAC are lower than those of $P F S / N A$ and POCAC, respectively, which verifies that CS can make better use of channel variation and serve more users than PFS.

2) MRR Violation Ratio: MRR violation happens when the achieved data rate of a user is less than its MRR in a scheduling period. MRR violation ratio here is defined as the total number of MRR violations divided by the number of successfully served users. MRR violation ratios with different

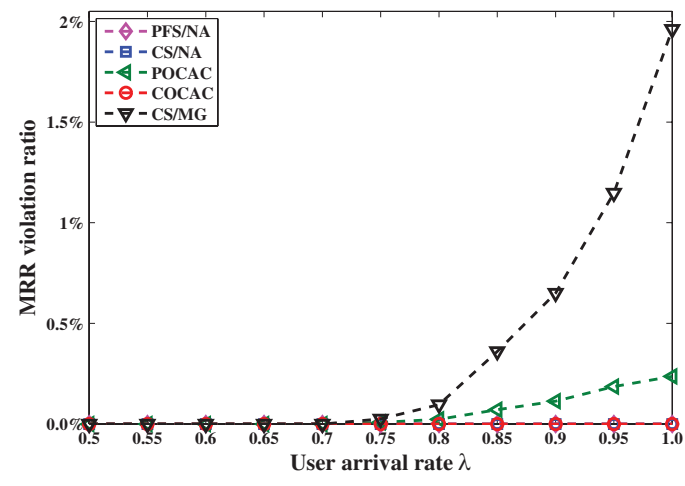

Fig. 2. MRR violation ratio.

user arrival rates are shown in Figure 2. We see that the MRR violation ratios of PFS/NA and CS/NA keep zero, because no MDG is considered in their CAC process and only conservative RRS is used for calculation of tokens. The MRR violation ratio of COCAC is also zero, even under an extremely high arrival rate, which is consistent with our objective, i.e., to serve as many users as possible with strict minimum rate guarantees. Since the MDG of PFS in POCAC is deduced from the assumption of i.i.d. channels, which is inaccurate in practical scenarios, the MRR violation happens when the user arrival rate is larger than 0.75 user/second. And the MRR violation ratio of $C S / M G$ is the highest, which indicates that all users' MRRs cannot be strictly guaranteed if we aggressively use the MDG in (6) which ignores the heterogeneous resource demand of each user to make the admission decision.

Through all above simulation results, we can find that our proposed COCAC algorithm can significantly reduce new call blocking rate, as well as strictly guarantee the minimum rate requirements of all serving users.

\section{CONCLUSIONS}

In this letter, we first deduced the MDG of CS and proposed a novel CAC algorithm with the consideration of minimum rate guarantee, namely, COCAC, which jointly uses CS and ORR for resource prediction and CAC decision. Then we conducted extensive simulation to demonstrate the superior performance of our COCAC algorithm.

\section{REFERENCES}

[1] P. Viswanath, D. N. C. Tse, and R. Laroia, "Opportunistic beamforming using dumb antennas," IEEE Trans. Inf. Theory, vol. 48, pp. 1277-1294, June 2002.

[2] T. Bonald, "A score-based opportunistic scheduler for fading radio channels," in Proc. European Wireless, 2004.

[3] D. Park, H. Seo, H. Kwon, and B. G. Lee, "Wireless packet scheduling based on the cumulative distribution function of user transmission rates," IEEE Trans. Commun., vol. 53, pp. 1919-1929, Nov. 2005.

[4] S. Patil and G. de Veciana, "Measurement-based opportunistic scheduling for heterogeneous wireless systems," IEEE Trans. Commun., vol. 57, no. 9, pp. 2745-2753, Sep. 2009.

[5] X. Wang, G. B. Giannakis, and A. G. Marques, "A unified approach to QoS-guaranteed scheduling for channel-adaptive wireless networks," Proc. IEEE, vol. 95, no. 12, pp. 2410-2431, Dec. 2007.

[6] S. S. Kulkarni and C. Rosenberg, "Opportunistic scheduling policies for wireless systems with short term fairness constraints," in Proc. IEEE Globecom, Dec. 2003, pp. 533-537.

[7] S. Patil and G. de Veciana, "Managing resources and quality of service in heterogeneous wireless systems exploiting opportunism," IEEE/ACM Trans. Netw., vol. 15, pp. 1046-1058, Oct. 2007. 